\title{
Spatial Structure of Emission Intensity in Capacitive RF Discharge of He:Ne Mixture at Moderate Pressures
}

\author{
Sh. Al-Hawat And M. Akel \\ Department of Physics, Atomic Energy Commission, Damascus, P.O. Box 6091, Syria
}

(Received December 03, 2009; in final form April 21, 2010)

\begin{abstract}
The spatial structure of emission intensity in a capacitive radio frequency discharge at $13.56 \mathrm{Mhz}$ in He:Ne mixture with total pressures of more than hundreds of Pascal was studied using the optical emission spectroscopy technique. The spectral line distributions at the axis of the discharge gap vs. the distance between two plane aluminium electrodes were found for: the line of $585.2 \mathrm{~nm}(\mathrm{Ne})$ and $447.15 \mathrm{~nm}(\mathrm{He})$ in 5:1 ratio of the mixture at pressure of $800 \mathrm{~Pa}$ for different driving powers 10,20 , and $30 \mathrm{~W}$; the line of $585.2 \mathrm{~nm}$ in the range of $733-4393 \mathrm{~Pa}$ at power of $20 \mathrm{~W}$ in 10:1 ratio of the mixture; lines of $632.8+633.4 \mathrm{~nm}(\mathrm{Ne})$ at ratios of 5:1, 10:1, 15:1, a power of $20 \mathrm{~W}$, under pressure of $1400 \mathrm{~Pa}$; lines of $585.2 \mathrm{~nm}, 632.8+633.4 \mathrm{~nm}, 640.2 \mathrm{~nm}$, and $703.2 \mathrm{~nm}(\mathrm{Ne})$ and $447.15 \mathrm{~nm}, 706.52 \mathrm{~nm}(\mathrm{He})$, at a pressure of $1467 \mathrm{~Pa}$ and a power of $20 \mathrm{~W}$ in ratio 10:1. Under chosen operational conditions, the measured relative spectral intensities for all studied lines in the middle of the discharge gap show the existence of the $\alpha$-regime in the RF discharge. The dc-bias voltage $v s$. the total pressure and the dc-bias voltage--power characteristics were obtained at certain conditions.
\end{abstract}

PACS numbers: 52.80.Pi

\section{Introduction}

The steady interest in capacitive radio-frequency (CRF) discharges is still arising due to the variety of their applications in material processing, microelectronics industry and laser technology. Moreover, the CRF discharge at moderate pressures is widely used in a lot of various practical applications, such as gas lasers and plasma -chemical reactors, and a great progress has been achieved in this area.

Therefore, such applications create a wide range of fundamental and practical investigations, and require a good understanding of all aspects of the plasma behavior under various operating conditions [1-4].

It is well known that, in the CRF discharges - depending on the operating conditions - there are two distinguished regimes, a low-current $\alpha$ regime and a high-current $\gamma$ regime. In both regimes the plasma electrons or the secondary electrons emitted from the electrodes play a dominant role in the energy deposition mechanisms. In the $\alpha$ regime, electrons acquire energy from the oscillation motion of the sheath for excitation and ionization of gas atoms in the bulk plasma. Whereas in the $\gamma$ regime - when the discharge power is high enough and if secondary electron emission is present - most of the energy deposition is due to electrons emitted by the electrodes and accelerated in the sheaths. The transition between these two regimes is dependent on the applied voltage, the kind and pressure of the gas $[5,6]$. In the present study, a particular consideration will be given to emphasis $\alpha$ regime as such discharges which are suitable for RF gas lasers [7].

The electrical characteristics (voltage and current density) of CRF discharges at moderate pressures for both modes vs. the length between two electrodes have been investigated previously [2]. Meanwhile, there is a lack in the spectroscopic investigations for spatial structure of CRF discharges in He:Ne mixture under moderate pressures. For more understanding of the physical nature of processes involved in the sheath and glow parts in CRF discharges, it is necessary to study the spatial distribution of spectral lines intensities of gases involved in such discharges $[3,8]$.

The spatial profiles of spectral lines intensities in CRF discharges could be obtained using, optical emission spectroscopy (OES), which has proved to be the most useful technique. The spatial emission profiles of the discharge could be served efficiently to get information on the kinetics of ionization and excitation in CRF discharges [9-14]. Also, they could provide information about the width of the sheath and the length of the bulk plasma depending on the operating conditions and the excitation potential of each spectral line.

To model the CRF discharge, we need experimental data about the features of RF discharge in various conditions, for instance, a spatial structure and spectral parameters of the planar CRF discharge in gas mixtures such as $\mathrm{CO}_{2}: \mathrm{N}_{2}: \mathrm{He}$ and $\mathrm{He}: \mathrm{Ne}$ are not satisfactory investigated [4]. 
For this purpose, measurements concerning the axial distribution of spectral intensity for lines from $\mathrm{Ne}$ and He spectra in a capacitively coupled parallel-plate $R F$ discharge at $13.56 \mathrm{MHz}$ in He:Ne mixture using a spatially resolved optical emission spectroscopy will be performed. These measurements will be carried out with pressure varied between 400-4400 $\mathrm{Pa}$ and power between 10-30 W for ratios 5:1, 10:1, 15:1 of He:Ne mixture.

The registration of spectral line intensities will be provided by scanning the whole $\mathrm{RF}$ discharge gap between two aluminum parallel planar electrodes. All of these are for analyzing the effect of parameters such as driving rf power, total pressure of the mixture and gas composition on the transitions intensity in He:Ne mixture for different lines from $\mathrm{He}$ and Ne spectra.

\section{Experimental}

The experimental apparatus scheme which was used is shown in Fig. 1, it consists of the following parts:

1. A high vacuum discharge chamber taken from Pyrex with dimensions of $12 \mathrm{~cm}$ length, and $4 \mathrm{~cm}$ internal diameter, which is provided by two aluminium parallel-plates serving as electrodes, each of them is with $3.4 \mathrm{~cm}$ length, $2.5 \mathrm{~cm}$ width and $0.2 \mathrm{~cm}$ thickness. In the evacuated state the aluminum plates are separated by a gap of $1 \mathrm{~cm}$;

2. Optical components, which include a collecting lens and a rotating plane-parallel plate with a thickness of $5 \mathrm{~cm}$;

3. An optical emission spectroscopy system which includes: a monochromator, a photomultiplier, a spectro-radiometer, and accessories [15];

4. A radio-frequency generator with a frequency of $13.56 \mathrm{MHz}$, and power of $1000 \mathrm{~W}$, a matching box which is used to perform impedance matching between the RF generator and the discharge chamber;

5. A vacuum system includes rotary and diffusion pumps and it is provided with Pirani and Penning vacuum meters; a gas supplier with two $\mathrm{He}$ and $\mathrm{Ne}$ cylinders and valves. The system can be evacuated to a base pressure of $1 \times 10^{-3} \mathrm{~Pa}$.

The discharge voltage is provided by a $\mathrm{RF}$ generator and a matching box. During the good matching, most of driving power goes to dissipate into the discharge, and the reflected power should have a negligible value. The applied power value is taken from the readout of the RF generator during the discharge, and it was not measured directly. In addition to that, the dc-bias voltage applied on the discharge is also given by the generator itself. Unfortunately the RF discharge voltage was not measured during the experiment.

We have recorded the spatial distribution of a spectral intensity in various compositions of gas mixture by

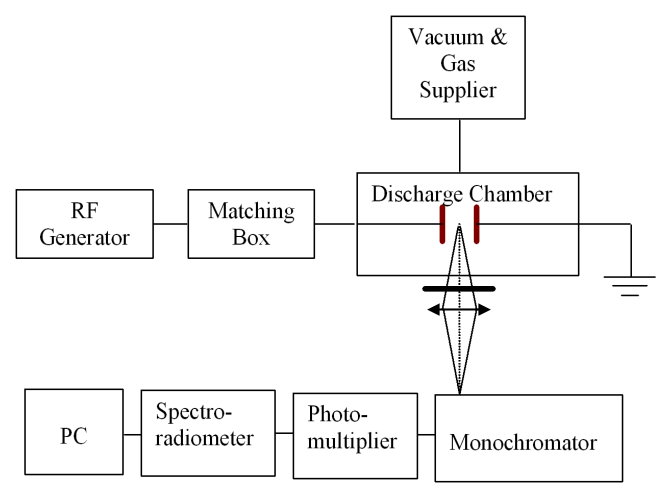

Fig. 1. Schematic diagram of experimental apparatus.

scanning the whole discharge along the transversal gap between two electrodes. The experiments were carried out on He:Ne mixture with ratios 5:1-15:1, with total pressure $p=400-4400 \mathrm{~Pa}$, the dissipated power in the discharge $P \leq 30 \mathrm{~W}, \mathrm{RF}$ voltage was applied to one electrode, whereas the other one was grounded.

The scheme of a power supply ensured a possibility to excite a volumetric stable capacity discharge in mixture He:Ne at mentioned conditions, the dc-bias voltage drop on the electrodes during the experiment was less than $80 \mathrm{~V}$.

A structure of CRF discharge was determined by the registration of an emission spectral intensity of selected lines from Ne and He spectra. The optical features of the discharge were measured using an automated monochromator (Bentham M300BA) with a photomultiplier (PM, Bentham DH-2) [15].

This setup allows scanning the whole gap between the electrodes by using a rotating parallel glass plate through a time interval of 20-30 s. The axial scan method was used to study the discharge between electrodes recording the intensity distribution at different spectral lines.

The spectral lines examined from neon are:

$$
\begin{aligned}
& 585.2 \mathrm{~nm}\left(3 p\left({ }^{1} S_{0}\right)(18.97 \mathrm{eV}) \rightarrow 3 s\left({ }^{1} P_{1}^{0}\right)\right), \\
& 640.2 \mathrm{~nm}\left(3 p\left({ }^{3} D_{3}\right)(18.56 \mathrm{eV}) \rightarrow 3 s\left({ }^{3} P_{2}^{0}\right)\right), \\
& 703.2 \mathrm{~nm}\left(3 p\left({ }^{3} S_{1}\right)(18.38 \mathrm{eV}) \rightarrow 3 s\left({ }^{3} P_{2}^{0}\right)\right), \\
& 633.4 \mathrm{~nm}\left(3 p\left({ }^{3} D_{2}\right)(18.57 \mathrm{eV}) \rightarrow 3 s\left({ }^{3} P_{2}^{0}\right)\right), \\
& 632.8 \mathrm{~nm}\left(5 s\left(3 s_{2}\right)(20.66 \mathrm{eV}) \rightarrow 3 p\left(2 p_{4}\right)\right),
\end{aligned}
$$

where the excitation of the level $(3 s)$ in neon for laser action occurs in two steps [16]:

$$
\begin{gathered}
e^{-}+\mathrm{He} \rightarrow e^{-}+\mathrm{He}_{\text {met }}^{*}\left(2^{1} S\right), \\
\mathrm{He}_{\text {met }}^{*}+\mathrm{Ne} \rightarrow \mathrm{He}+\mathrm{Ne}^{*}(3 s) .
\end{gathered}
$$

and the spectral lines examined from helium spectrum are:

$$
\begin{aligned}
& 447.15 \mathrm{~nm}\left(4^{3} D(23.73 \mathrm{eV}) \rightarrow 2^{3} P\right), \\
& 706.52 \mathrm{~nm}\left(3^{3} S(22.71 \mathrm{eV}) \rightarrow 2^{3} P\right) .
\end{aligned}
$$


The axial spectral measurements are based on the focusing of the light emitted from the discharge with the help of a lens on an entering slot of monochromator, which will be processed by the photomultiplier and a programmable current amplifier, to be displayed on a PC computer.

The spatial resolution of this arrangement is better than $0.25 \mathrm{~mm}$, the spectral resolution of the monochromator is $0.75 \mathrm{~nm}$ and the relative error of the spectral intensities by this method is about $15 \%$.

\section{Results and discussion}

The obtained results concerning the optical emission profiles for different lines from He and Ne spectra in the He:Ne mixture with power, total pressure and composition $v s$. the distance between the electrodes are shown in Figs. 2-5. The whole measurements are related to the discharge axis and the optical emission spectroscopy.

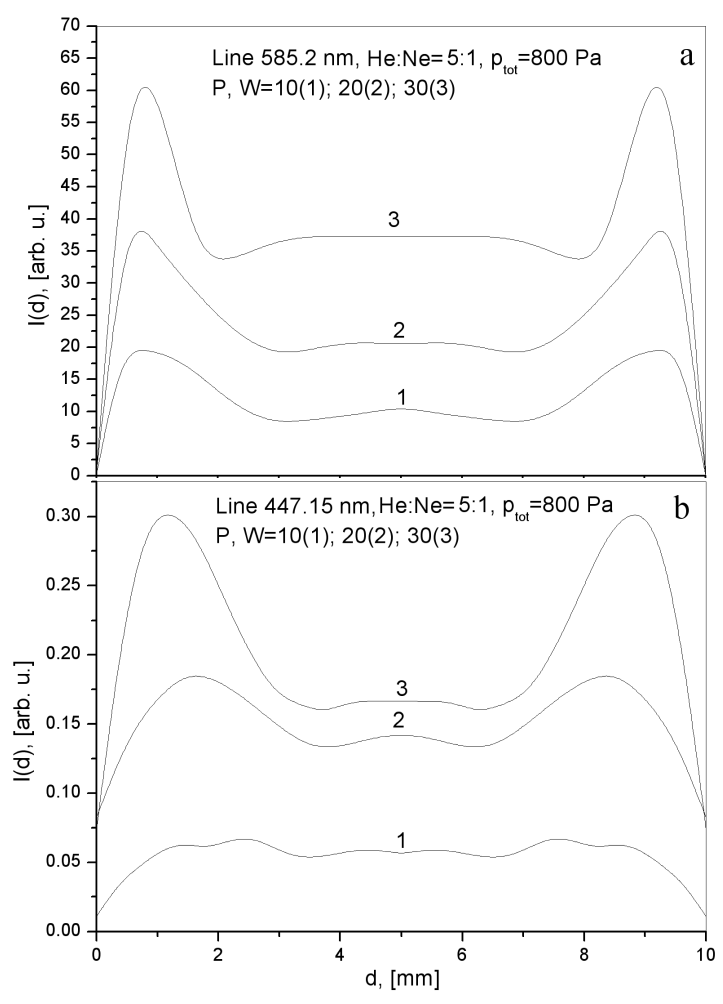

Fig. 2. The axial distribution of spectral intensities of (a) $585.2 \mathrm{~nm}(\mathrm{Ne})$ and (b) $447.15 \mathrm{~nm}(\mathrm{He})$ at total pressure $800 \mathrm{~Pa}$ and He:Ne mixture ratio of $5: 1$ for power variation between 10 and $30 \mathrm{~W}$.

As seen from Fig. 2a,b, with the power increasing, the intensity of plasma emission is increased. In general, the axial spectral intensity of the lines $585.2 \mathrm{~nm}$ and $447.15 \mathrm{~nm}$ increases by increasing the driving $\mathrm{RF}$ power from $10 \mathrm{~W}$ to $30 \mathrm{~W}$ at a total pressure of $800 \mathrm{~Pa}$ of a ratio of $5: 1$. This finding is attributed to the increase of electron density in the discharge; while the sheath

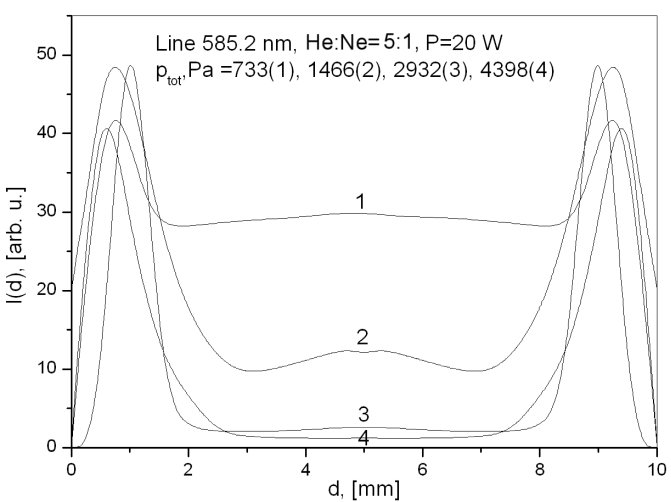

Fig. 3. The axial distribution of spectral intensities of $585.2 \mathrm{~nm}(\mathrm{Ne})$ at power of $20 \mathrm{~W}$, total pressure 733-4400 $\mathrm{Pa}$ of He:Ne mixture with ratio of 10:1.

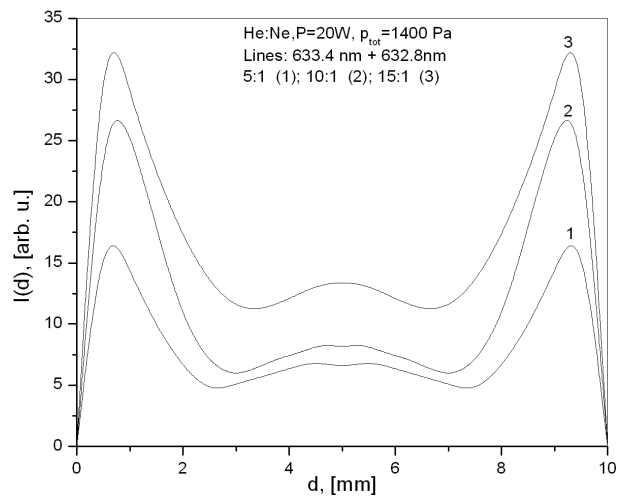

Fig. 4. The axial distribution of spectral intensities of $633.4 \mathrm{~nm}+632.8 \mathrm{~nm}$ from Ne for 5:1, 10:1 and 15:1 of $\mathrm{He}: \mathrm{Ne}$ at a total pressure of $1400 \mathrm{~Pa}$, and a power of $20 \mathrm{~W}$.

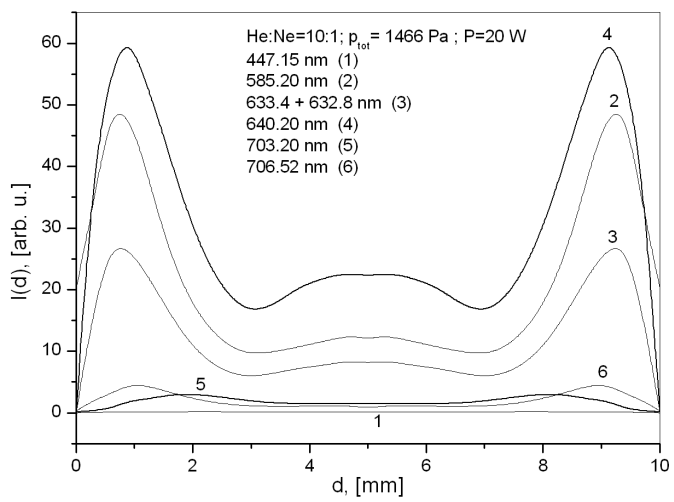

Fig. 5. The axial distribution of spectral intensities of $703.2 \mathrm{~nm} ; 640.2 \mathrm{~nm} ; 633.4 \mathrm{~nm}+632.8 \mathrm{~nm} ; 585.2 \mathrm{~nm}$ from Ne spectrum and $706.52 \mathrm{~nm} ; 447.15 \mathrm{~nm}$ from He spectrum at total pressure $1466 \mathrm{~Pa}$; ratio of $10: 1$ and power $20 \mathrm{~W}$. 
thickness is decreasing. One can predict from these distributions that in the middle part of the discharge, the main contribution to the total current is provided by the electrons, whereas near the electrodes, the displacement current prevails. As the driving power increases, the electric field near the electrodes also increases, thus insuring a higher displacement current, which is proportional to $\partial E / \partial t$ (where $E$ is the electric field). The increase in the reduced electric field $E / N$ (where $N$ is the gas mixture density) near the electrodes causes an increase in the excitation and ionization rates and consequently the spectral intensity $[7,8]$.

No significant difference in the behavior of these two lines (Ne: $585.2 \mathrm{~nm}$ and He: $447.2 \mathrm{~nm}$ ) as well as for the other observed lines was observed and therefore they are not presented here.

Figure 3 shows that there is a tendency to get a slight narrowing of the sheath width with increasing the pressure, at a constant power, while the intensity is decreasing especially in the middle part of the discharge. Intensity decrease in the "bulk" can be expected, as, at fixed power, with increase of the pressure, electron mean free path decreases i.e. electrons have less chance to gain enough energy between collisions to perform excitation and ionization. As the pressure increases from 733 to $4398 \mathrm{~Pa}$ at 10:1 ratio, the spectral intensity of line $585.2 \mathrm{~nm}$ decreases from 30 to 1 arb. unit. This may be occurred due to the decreasing of $E / N$ values in the middle of the discharge gap. Thus, it indicates a tendency of the electric field to arise inside the sheath. This effect is related to the fact that the $\mathrm{rf}$ discharge voltage is combined from the voltage drops across the plasma column and electrode sheaths $[2,16]$.

In addition, the intensity of the central glow part of the discharge is much lower than that observed in the electrode sheath. This effect could be connected with the preferential spatial localization of the processes of electron energy losing in the electron-atom inelastic collisions. It is observed that, with an increase in pressure the positions of the peaks are slightly shifted closer to the electrodes. Our data are congruent with results presented in [9].

However, one can observe in a range of $733<p<$ $4398 \mathrm{~Pa}$, that the intensity $I(d)$ poorly depends on pressure inside the sheaths, but it decreases with increasing the pressure in the bulk plasma or in the central part of the discharge. The sizes of both zones practically do not vary so much with the pressure growth.

On the other hand, the increasing of He content in a mixture He:Ne from 5 to 15 at a total pressure of $1400 \mathrm{~Pa}$, as indicated in Fig. 4, causes a growth of the intensity in the sheaths but their widths are slightly varied.

The influence of He increasing ratio from 5:1 to 15:1 on transitions of $632.8 \mathrm{~nm}+633.4 \mathrm{~nm}$ in Ne spectrum is clearly seen here. But this could be useful for the case of He:Ne lasers.
As it is shown in Fig. 5, the spatial distribution of the spectral intensity is different according to the excitation potential of each spectral line, and the variation of spectral intensity has a similar behavior for all studied lines.

It could be noticed that the profiles are almost perfectly symmetric, which demonstrate that the discharge itself is symmetric. The emitted intensity grows with increasing power and as it is seen from Fig. 6a, that the intensity at the centre of the discharge $I_{\mathrm{m}}$ slightly increases, even not proportionally, in comparison to the maximum intensity in the sheath $I_{s}$ for all studied lines.
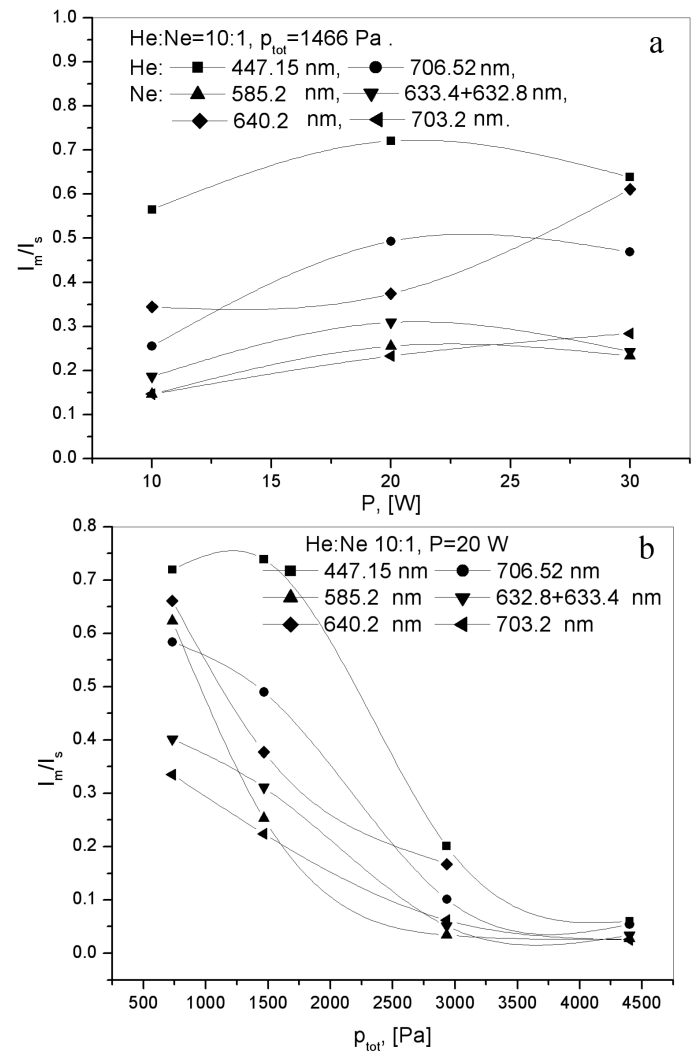

Fig. 6. The ratio of spectral intensities in the bulk plasma and the electrode sheath vs. (a) the power at certain pressure of $1466 \mathrm{~Pa}$ and vs. (b) the total pressure for different lines and power of $20 \mathrm{~W}$ with ratio of $10: 1$.

The experimental results show that the discharge in the glow plasma remained in $\alpha$ regime in the given power range, as the power is not sufficient to transfer the discharge to the $\gamma$ regime. Figure 6 a shows, that the ratio of spectral intensities is varying slowly with the increase of the applied RF power; this indicates that the generated phase has $\alpha$ type. While Fig. $6 \mathrm{~b}$ gives the variation of the relative intensity $v s$. the total pressure at certain power of $20 \mathrm{~W}$.

These results show that OES is a diagnostic technique which could give a wide range of spectral data. For example, absolute intensities may be associated with absolute excitation rates, and the most importantly is the spa- 
tially resolved excitation profiles provide information on spatial extent of the regions of high energy particles that sustain the plasma.

The effect of power, pressure and mixture composition on the axial distribution of the spectral emission from the plasma was studied. For example at low powers these distributions were symmetric and had two sheaths in the vicinity of the electrodes.

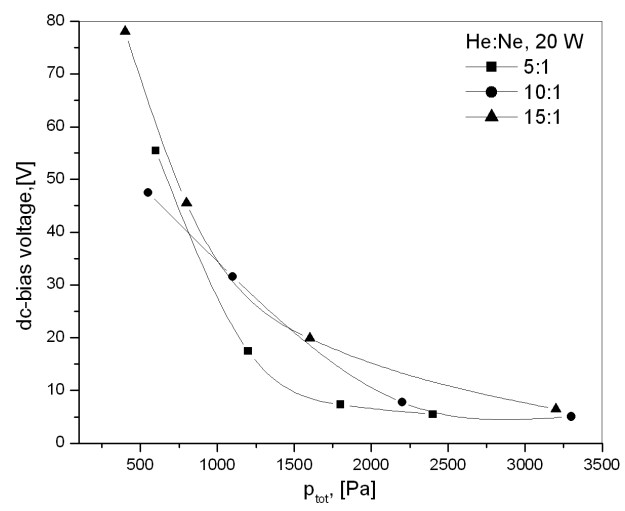

Fig. 7. The applied dc-bias voltage vs. the total pressure at $20 \mathrm{~W}$ and ratios of $5: 1 ; 10: 1 ; 15: 1$.

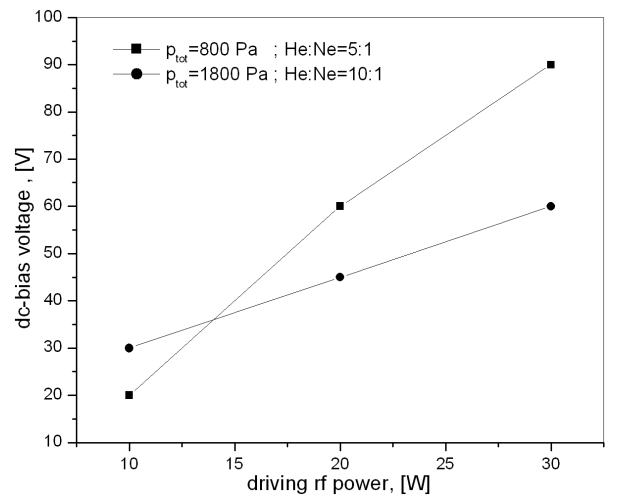

Fig. 8. The applied dc-bias voltage $U_{\text {dc-bias }} v s$. the driving rf power for the mixture $\mathrm{He}: \mathrm{Ne}=5: 1$ and 10:1 at total pressures $p=800 \mathrm{~Pa}$ and $p=1800 \mathrm{~Pa}$, respectively.

From the readout of generator, some results about the dc-bias voltage and the applied RF power were obtained. For instance, Fig. 7 gives the applied dc-bias voltage $v s$. the total pressure for three ratios of He:Ne mixture at power of $20 \mathrm{~W}$, while Fig. 8 represents the applied dc-bias voltage versus the driving $\mathrm{RF}$ power for two different compositions of gas mixture $\mathrm{He}: \mathrm{Ne}=5: 1$ and 10:1 at pressure of $p=800 \mathrm{~Pa}$ and $p=1800 \mathrm{~Pa}$, respectively.

Concerning the width of the sheath, in accordance with the elementary theory of CRFD [2], the width is almost equal to the amplitude $\Delta_{\mathrm{m}}$ of the electron oscillation at the RF field:

$$
\Delta_{\mathrm{m}}=e E / m \nu \omega,
$$

where $e$ and $m$ are charge and mass of electron, $\nu$ is the effective number of electron collisions and $\omega$ is the circular frequency of the applied RF voltage.

As one could see from Eq. (1), the calculated width of the sheath varies in inverse proportion to pressure of gas. According to our measurements such dependence is not so strong (see Fig. 3).

The width of the sheaths, where lasing is impossible, puts a special limit on the transversal dimensions of laser active elements L, which it could be at least:

$$
L>2 \Delta_{\mathrm{m}} \text {. }
$$

For example, most of modern $\mathrm{CO}_{2}$ lasers are designed under these conditions Eqs. (1)-(2) [17].

\section{Conclusions}

In conclusion, the emission profiles are typical for an $\alpha$-CRF discharge with two sheaths in the vicinity of electrodes. The visible emission intensity in the central part of the discharge is much lower than that observed near the electrodes.

The effect of plasma emission localization in a neighborhood of electrodes could be connected with the preferential spatial localization of the processes of electron energy losing in the electron-atoms inelastic collisions. Near the electrodes, both the ionization and excitation of atoms in a discharge mainly happen by a field of an uncompensated positive charge in the sheaths.

The existence of two sheaths near the electrodes, which differs from the glow region in the central part of discharge, is one of the most interesting feature of an $\alpha$ phase CRF discharge applicable for gas lasers.

For the plasma-chemical reactors, it is a very promising region, where a lot of ionic processes are very intensive. On the contrary, for gas lasers, these regions are non-working and could be as narrow as possible, the lasing could exist only in a region between two boundary sheaths. However in both cases it is necessary to determine the real sheath width for any plasma device optimization.

\section{Acknowledgments}

Authors would like to thank General Director of AECS for his continuous encouragement, guidance and support.

Many thanks to the staff of RF plasma discharge laboratory for their help and efforts in the experiment.

Also, many thanks to L.N. Orlov and V. Necrashevich from Physics Institute in Minsk, Belarus Academy of Sciences for their cooperation in this work.

\section{References}

[1] D. Hall, C. Hill, in: Handbook of Molecular Lasers, Ed. P.K. Cheo, Dekker Inc., New York 1987, Ch. 3.

[2] Y.P. Raizer, M.N. Shneider, N.A. Yatsenko, Radio-Frequency Capacitive Discharge, CRC Inc. London, 1995, Ch. 1,2 . 
[3] A. Melzer, R. Flohr, A. Piel, Plasma Sources Sci. Technol. 4, 424 (1995).

[4] V.V. Azharonok, I.I. Filatova, V.D. Shimanovich, V.N. Ochkin, L.N. Orlov, J.I. Necrashevich, V.V. Nevdakh, Lebedev Physical Institute, Preprint 37, (2003).

[5] Ph. Belenguer, J.P. Boeuf, Phys. Rev. A 41, 4447, (1990).

[6] V.A. Godyak, R.B. Piejak, B.M. Alexandrovich, Phys. Rev. Lett. 68, 40 (1992).

[7] P.P. Vitruk, H.J. Baker, D.R. Hall, J. Phys. D; Appl. Phys. 25, 1767 (1992).

[8] S.A. Starostin, K.J. Boller, P.J. Peters, Y.B. Udaloo, I.V. Kochetov, A.P. Napartovich, Plasma Phys. Rep. 28, 68 (2002).

[9] I. Odrobina, M. Kando, Plasma Sources Sci. Technol. 5, 517 (1996)

[10] Z.Lj. Petrovic, S. Bzenic, J. Jovanovic, S. Djurovic, J. Phys. D; Appl. Phys. 28, 2287 (1995).
[11] M.A. Lieberman, A.J. Lichtenberg, Principles of Plasma Discharges and Materials Processing, Wiley, New York 1994.

[12] V.A. Godyak, R.B. Piejak, N.A. Sternberg, IEEE Trans. Plasma Sci. 21, 378 (1993).

[13] M.M. Turner, Phys. Rev. Lett. 75, 1312 (1995).

[14] S.V. Berezhnoi, I.D. Kaganovich, M. Misina, A. Bogarets, R. Gijbels, IEEE Trans. Plamsa Sci. 27, 1339 (1999).

[15] S. Al-Hawat, IEEE Trans. Plasma Sci. 28, 1682 (2000).

[16] A. Bogaerts, E. Neyts, R. Gijbels, J. van der Mullen, Spectrochim. Acta B 57, 609 (2002).

[17] L.N. Orlov, V.V. Azharonok, J. Appl. Spectrosc. 66, 826 (1999). 